\title{
Transferable micromachined piezoresistive force sensor with integrated double-meander-spring system
}

\author{
Gerry Hamdana $^{1,2}$, Maik Bertke ${ }^{1,2}$, Lutz Doering ${ }^{3}$, Thomas Frank ${ }^{4}$, Uwe Brand ${ }^{3}$, \\ Hutomo Suryo Wasisto ${ }^{1,2}$, and Erwin Peiner ${ }^{1,2}$ \\ ${ }^{1}$ Institute of Semiconductor Technology (IHT), Technische Universität Braunschweig, Hans-Sommer-Straße \\ 66, 38106 Braunschweig, Germany \\ ${ }^{2}$ Laboratory for Emerging Nanometrology (LENA), Langer Kamp 6a, 38106 Braunschweig, Germany \\ ${ }^{3}$ Department 5.1 Surface Metrology, Physikalisch-Technische Bundesanstalt (PTB), Bundesallee 100, \\ 38116 Braunschweig, Germany \\ ${ }^{4}$ CiS Forschungsinstitut für Mikrosensorik GmbH, Konrad-Zuse-Straße 14, 99099 Erfurt, Germany
}

Correspondence to: Gerry Hamdana (g.hamdana@tu-bs.de)

Received: 30 September 2016 - Revised: 9 February 2017 - Accepted: 9 February 2017 - Published: 2 March 2017

\begin{abstract}
A developed transferable micro force sensor was evaluated by comparing its response with an industrially manufactured device. In order to pre-identify sensor properties, three-dimensional (3-D) sensor models were simulated with a vertically applied force up to $1000 \mu \mathrm{N}$. Then, controllable batch fabrication was performed by alternately utilizing inductively coupled plasma (ICP) reactive ion etching (RIE) and photolithography. The assessments of sensor performance were based on sensor linearity, stiffness and sensitivity. Analysis of the device properties revealed that combination of a modest stiffness value (i.e., $(8.19 \pm 0.07) \mathrm{N} \mathrm{m}^{-1}$ ) and high sensitivity (i.e., (15.34 \pm 0.14$\left.) \mathrm{V} \mathrm{N}^{-1}\right)$ at different probing position can be realized using a meander-spring configuration. Furthermore, lower noise voltage is obtained using a double-layer silicon on insulator (DL-SOI) as basic material to ensure high reliability and an excellent performance of the sensor.
\end{abstract}

Rapid development of micro-/nano-fabrication techniques in the past years has had a significant impact not only on higher integration density of the micro-/nano-components leading to enhanced device performance but also on the need of better and more precise quality control of the individual component itself. Consequently, more precise characterizations of physical properties in smaller scale (e.g., micro- or nanoscale) and their know-how are in demand.

As one of the fundamental techniques in material characterization, nano-indentation has already been able to perform measurements at a resolution down to $1 \mathrm{nN}$ with a minimum displacement of $<1 \mathrm{~nm}$. During measurement, this method applies certain loads to create indentation patterns on the sample, which can be used to determine hardness (Fu et al., 2015; Li and Brand, 2013; Nili et al., 2013; Yetna Njock et al., 2016). However, to date there is still no transferable standard available, which can be used for calibrating nanoindentation instruments in the micro- and nano-range. For this purpose, a system for calibrating very small forces is therefore needed. Although different approaches (e.g., piezoelectric: Choi et al., 2016; Lee et al., 2014; Mohan et al., 2015; capacitive: Brand et al., 2016; Viry et al., 2014; Woo et al., 2014; and magnetic flux change: Mehrtash et al., 2015) have been used to realize small force scales. Measuring forces in small scales is also very useful for robotics and medical engineering (e.g., a silicon on insulator (SOI)-based piezoresistive sensor was attached on the tip of the guidewire to detect motions in $z$ axis during the catheterization process) while connecting the micro- and macro-environments is possible by closed-loop disturbance compensation of the system (Mizutani, 2013, 2014; Ousaid et al., 2015).

Micro-fabricated silicon-based force standards for calibrating hardness testing instruments were described in detail elsewhere and are commercially available from Simet- 

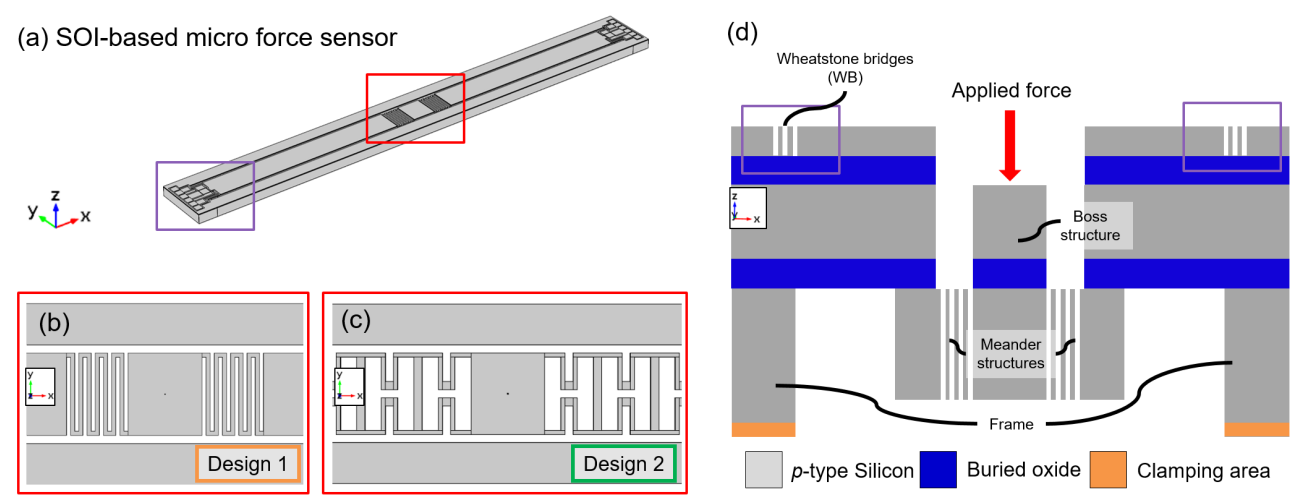

Figure 1. Three-dimensional (3-D) model of proposed SOI-based micro force sensor (a). Magnified top view of different spring designs (b, c). Cross sectional view of the micro force sensor based on design 1 showing the point loading on the probing area (boss) and the fixing of the sensor to the device holder (d).
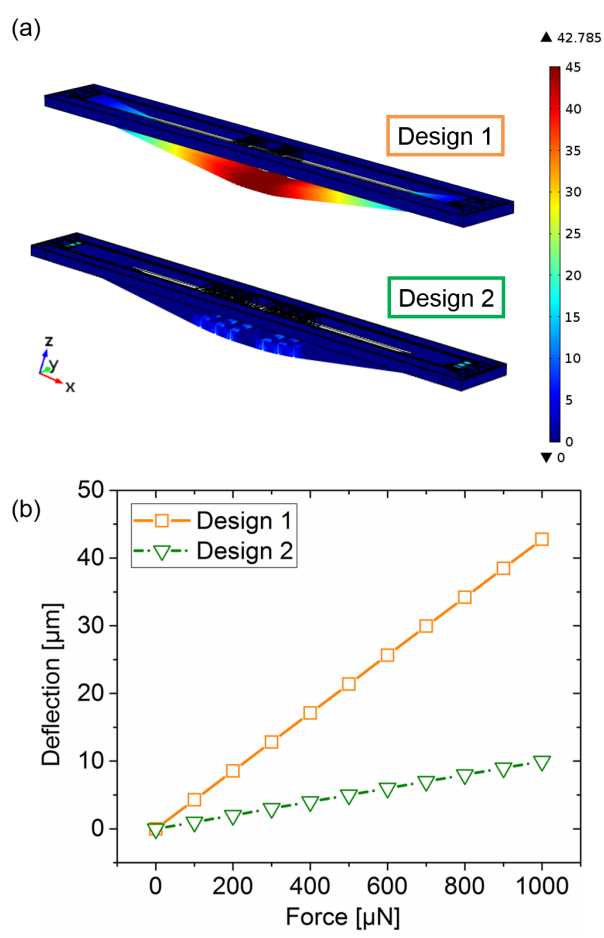

Figure 2. Simulated 3-D model of both device configurations (i.e., designs 1 and 2). The color legend shows deflection state on the boss structure in $\mu \mathrm{m}$ (a). The sensor displacement in $z$ direction was computed with a maximum force of $1000 \mu \mathrm{N}(\mathbf{b})$.

rics GmbH, Germany (Frühauf et al., 2007a, b). However, these artifacts do not offer a direct self-sensing output. Furthermore, they have rather large stiffness values (i.e.,

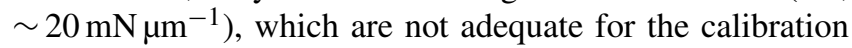
procedure of indentation instruments in the $\mu \mathrm{N}$ range. In micro-electromechanical system (MEMS) technology, several transduction mechanisms are available, which can be taken into consideration for the design of a self-sensing force artifact. Among them, capacitive, optical, piezoelectric and piezoresistive approaches are most popular. With capacitive sensors, the available measurement range could be limited by the small gap (representing the capacitance) between the probing area of the force sensor and the bottom plate. Measuring small deflections optically is only possible on a reflective surface. Fabrication of an additional reference structure on the probing area and specialized instrumentation are needed to perform reliable deflection measurements. The use of piezoelectric transduction requires deposition of a piezoelectric layer on the spring beam, which may induce residual stress. In contrast, piezoresistive strain gauges can be directly realized using doped silicon resistors; i.e., different robust designs of force sensors are possible by combining standard silicon processing steps.

Recently, a force artifact for the micro-Newton range based on double-sided clamped probing body via combined meander-membrane springs (Doering et al., 2013) were proposed. Meander-type springs in combination with bending springs are well suited to take up the lateral strain, which increases with the deflection of the probing body and are thus able to provide a large linear range of the micro force artifact. Samples of this artifact, which were realized using reactive ion etching (RIE) at cryogenic temperature in silicon, confirm the load-deflection behavior derived from finite element modeling (FEM; Hamdana et al., 2016; Wasisto et al., 2015a). For an application under industrial conditions the micro force artifact comprises a strain-sensing piezoresistive Wheatstone bridge (WB) of very low cross-sensitivity to non-constant ambient conditions such as temperature, humidity and light. Furthermore, noise of the WB limiting the minimally detectable force should be as low as possible. For both low cross-sensitivity and noise, electrical and thermal decouplings of the WB from the membrane spring using a buried $\mathrm{SiO}_{2}$ layer were proven to be effective (Kähler et al., 2012, 2013). Utilizing this approach, we aim to fulfill the need of small force standards; e.g., for nano-indenters fol- 
lowing specifications for small force calibration procedure are required (Table 1).

In this paper, we assess the performance of our fabricated force sensors that have double-meander-spring structures and compare them to industrially manufactured sensor devices. First, mechanical and electrical properties of both designs were numerically analyzed. Afterwards, sensor fabrication was carried out using inductively coupled plasma (ICP) deep reactive ion etching (DRIE) at cryogenic temperature as the key process (Wasisto et al., 2014; Merzsch et al., 2014). Direct-sensing capability of different types of piezoresistive strain gauges (i.e., etched and implanted WBs) was carried out. Furthermore, point-force applications at different positions along the $x$ axis of the boss structure were carried out to evaluate the sensor performance under conditions corresponding to the typical nano-indenter force calibration procedure.

\section{Design and simulation}

In this paper, we aim to evaluate the performance of our developed micro force sensor (i.e., design 1) in comparison with the fabricated device from the industry (i.e., design 2). The first step was to model accurate three-dimensional (3-D) representations of both micro force sensors and determine their physical properties (i.e., stiffness and sensitivity) in a finite element simulation using COMSOL Multiphysics 4.3b (COMSOL Multiphysics, 2013). In general, the model geometry was adapted to the actual chip size of the device, i.e., $20 \mathrm{~mm} \times 2.2 \mathrm{~mm}$. The sensor geometry consists of a probing area (i.e., boss structure), two meander-beam spring structures and two piezoresistive strain gauge elements (WBs) located at both clamped ends of the device (Fig. 1a). Two designs of the sensor were investigated by employing different spring structure configurations. Design 1 applies deep meander structures perpendicular to the $x-y$ plane with small regular gaps between the structures (Fig. 1b), while design 2 adopts a micro-structure with various lateral dimensions and gaps between the structures (Fig. 1c). When a vertical force is applied on the center of the boss structure, a smooth bending of the beams followed by elastic material deformation and changes of resistance values on both WB structures occurred (Fig. 1d). During the simulation, several mesh sizes were used in order to reduce computational time without sacrificing results reliability. In order to replicate the real device conditions, anisotropic single crystal silicon was selected and the 3-D models were rotated by $45^{\circ}$ in the $x-y$ plane, allowing for simulations along $<110>$ crystal orientation (Bonev and Zlatanov, 2002; COMSOL Multiphysics, 2013; Hopcroft et al., 2010).

Numerical simulation of both spring configurations was performed by utilizing several predefined physics interfaces, i.e., solid mechanics and piezoresistivity, domain currents (pzrd). For the mechanical boundary conditions, the bot-
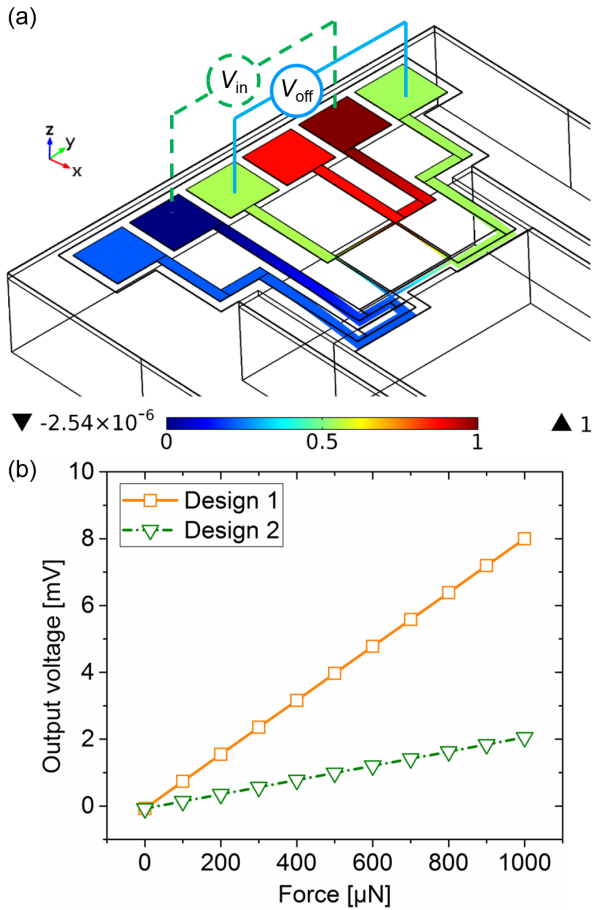

Figure 3. Simulated 3-D model of device sensitivity under an applied load of $1000 \mu \mathrm{N}$ (a). Sensor output voltage vs. vertical loads up to $1000 \mu \mathrm{N}$ (b).

tom part of the SOI-based micro force sensor was given a fixed motion constraint. In contrast, other parts of the models were set to be free. Moreover, the electrical potential boundary condition of $1 \mathrm{~V}$ was applied to the input electrode of WB, whereas diagonally opposite output electrode was grounded thereby creating a DC input voltage $\left(V_{\text {in }}\right)$. As a result, changes of the output voltage ( $\left.V_{\text {off }}\right)$ can be determined through the two remaining WB contacts.

The mechanical and electrical properties of both sensor configurations (design 1 and design 2) were investigated. To capture the behavior of the device in the envisaged application range, vertical forces between 0 and $1000 \mu \mathrm{N}$ with a step size of $100 \mu \mathrm{N}$ were used. Owing to symmetry considerations, in the following we consider only one of the two meander-beam springs of the sensor. We define the coordinate system origin of the $x$ axis to be located within the clamped area of the beam. The origin of the $z$ axis, where the deflection $z$ direction as a function of an applied force were given, corresponds to a neutral layer of the vertical beam deflection. Along this layer, the longitudinal stress equals to zero. FEM results of different spring configurations under vertical loading up to $1000 \mu \mathrm{N}$ are presented in Fig. 2a. Theoretically, the longitudinal stress $\sigma_{\mathrm{L}}$ in the [110] direction 
Table 1. Required specifications of small force calibration standards for nano-indenters.

\begin{tabular}{lrrrrrr}
\hline $\begin{array}{l}\text { Force } \\
\text { component }\end{array}$ & $\begin{array}{r}\text { Measurement } \\
\text { range }\end{array}$ & Resolution & $\begin{array}{r}\text { Resonance } \\
\text { frequency }\end{array}$ & Bandwidth & Chip size & Probing area \\
\hline$z$ & $0-1 \mathrm{mN}$ & $0.1 \mu \mathrm{N}$ & $>100 \mathrm{~Hz}$ & $10 \mathrm{~Hz}$ & $<20 \mathrm{~mm} \times 20 \mathrm{~mm}$ & $>1 \mathrm{~mm} \times 1 \mathrm{~mm}$ \\
\hline
\end{tabular}

can be calculated using the following expression (Park et al., 2010):

$\sigma_{\mathrm{L}}=\frac{12(l-x) z}{w t^{3}} F$

where $x$ and $z$ represent the positions on $x$ and $z$ axes from the system origin located at the clamping positions and the neutral layer of the membrane springs, respectively. In Eq. (1) the geometrical factors of the beam are given by the beam length $l$, width $w$ and thickness $t$ (Park et al., 2010). Subject to material properties, the stress $\sigma_{\mathrm{L}}$ can be described as a product of the elastic modulus $E$ and the longitudinal strain $\varepsilon_{\mathrm{L}}$ along the $x$ axis.

$\sigma_{\mathrm{L}}=E \varepsilon_{\mathrm{L}}$

At the same deflection state, the mechanical deformation on the WB structures depends on the membrane bending radius $r$, which can be determined by

$r=\frac{z}{\varepsilon_{\mathrm{L}}}$

Taking geometrical, material and bending properties into account, the force applied on the device can be derived from Eqs. (1)-(3) as

$F=\frac{w t^{3} E}{12 r(l-x)}$.

The relationship between the applied vertical load $\Delta F$ and its resulting deflection of the probing body (boss) $\Delta z$ is referred as device stiffness $k$ :

$k=\frac{\Delta F}{\Delta z}$.

Figure 2(b) shows the deflections obtained using FEM with different sensor designs. The vertically applied load on design 1 was incrementally increased and leading to a linear rise of the boss deflection. As a result, design 1 exhibits a stiffness of $\left(23.39 \pm 9 \times 10^{-9}\right) \mathrm{N} \mathrm{m}^{-1}$. In comparison, design 2 indicates a stiffer structure response than design 1 . In this case, a stiffness of $\left(100.18 \pm 5 \times 10^{-13}\right) \mathrm{N} \mathrm{m}^{-1}$ was obtained, which is a factor of $\sim 4.3$ higher than that of design 1. A higher stiffness value implicates the requirement of higher applied forces to deflect the structure, which in practical applications can be limited by the instrument or probe to be calibrated. Fine tuning during measurement of very small forces may be problematic since the output signal (i.e., resistance changes of the WB) depends strongly on bending mechanism of the membrane part of the spring. Hence, sensor sensitivity needs to be taken into consideration, which was numerically analyzed by FEM based on the resistance changes $(\Delta R / R)$ of the WB (Wasisto et al., 2015b):

$\frac{\Delta R}{R}=\sigma_{\mathrm{L}} \Pi_{\mathrm{L}}+\sigma_{\mathrm{T}} \Pi_{\mathrm{T}}$,

where $\sigma_{\mathrm{L}}, \sigma_{\mathrm{T}}, \Pi_{\mathrm{L}}$ and $\Pi_{\mathrm{T}}$ are mechanical stresses along longitudinal and transverse directions and piezoresistive coefficients for each direction, respectively. For the WB structures and their electrical contact regions, doping concentrations of $5 \times 10^{18}$ and $1 \times 10^{19} \mathrm{~cm}^{-3}$ are assumed, respectively. To calculate resistance changes of the WB, a supply voltage $\left(V_{\text {in }}\right)$ of $1 \mathrm{~V}$ was applied diagonally (Wasisto et al., $2015 \mathrm{c}$ ) and the output voltage ( $\left.V_{\text {off }}\right)$ was determined on the opposite diagonal of the resulting full bridge configuration consisting of four resistors (i.e., $R_{1}=R_{2}=R_{3}=R_{4}$ ) along $<110>$ and its transverse direction leads to following relationship between input and output:

$V_{\text {off }}=V_{\text {in }} \times\left(\frac{R_{2}}{R_{1}+R_{2}}+\frac{R_{3}}{R_{3}+R_{4}}\right)$.

Figure 3 a shows the distributed voltage value within WB under vertical applied force of $1000 \mu \mathrm{N}$ obtained by FEM. The relation between load $F$ and output voltage $V_{\text {off }}$ on different meander form is linear for both designs; i.e., we can define a sensitivity:

$S=\frac{\Delta V_{\text {off }}}{\Delta F}$.

Comparing the different spring configurations, we find by a factor of $\sim 4$ higher sensitivity value (i.e., $\left.\left(8.07 \pm 4.07 \times 10^{-6}\right) \mathrm{V} \mathrm{N}^{-1}\right)$ for design 1 than design 2 (i.e., $\left.\left(2.13 \pm 2.98 \times 10^{-7}\right) \mathrm{V} \mathrm{N}^{-1}\right)$. This result is correlated directly with the simulated results for stiffness. Considering both device properties (i.e., stiffness and sensitivity), design 1 provides a more flexible spring structure and higher sensitivity than design 2 , which should be better suited for calibrating nano-indenters in the range of small forces. 
Table 2. Averaged measured sensor calibration values of designs 1 and 2 at different positions on the boss along the longitudinal ( $x$ axis, long.) and transverse ( $y$ axis, trv.) directions.

\begin{tabular}{lrr|rr|rr}
\hline \multirow{2}{*}{ Sensor design } & \multicolumn{2}{c|}{ Stiffness $\left(\mathrm{N} \mathrm{m}^{-1}\right)$} & \multicolumn{2}{|c|}{ Force sensitivity $\left(\mathrm{V} \mathrm{N}^{-1}\right)$} & \multicolumn{2}{c}{ Bending sensitivity $\left(\mathrm{V} \mathrm{m}^{-1}\right)$} \\
\cline { 2 - 7 } & Loading & Unloading & Loading & Unloading & Loading & Unloading \\
\hline 1 (long.) & $8.17 \pm 0.08$ & $8.13 \pm 0.08$ & $15.35 \pm 0.18$ & $15.55 \pm 0.15$ & $126.06 \pm 2.25$ & $127.11 \pm 1.94$ \\
2 (long.) & $70.54 \pm 2.98$ & $70.59 \pm 2.17$ & $4.10 \pm 0.64$ & $4.10 \pm 0.64$ & $305.40 \pm 38.55$ & $303.54 \pm 38.65$ \\
1 (trv.) & $8.18 \pm 0.06$ & $8.23 \pm 0.07$ & $15.26 \pm 0.32$ & $15.42 \pm 0.17$ & $126.48 \pm 2.70$ & $126.09 \pm 1.52$ \\
2 (trv.) & $71.83 \pm 1.31$ & $71.49 \pm 1.21$ & $4.19 \pm 0.49$ & $4.18 \pm 0.49$ & $311.64 \pm 33.47$ & $309.68 \pm 33.87$ \\
\hline
\end{tabular}

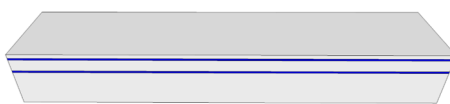

(a)

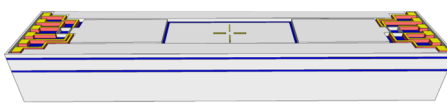

(d)

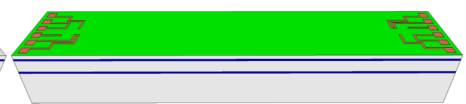

(b)

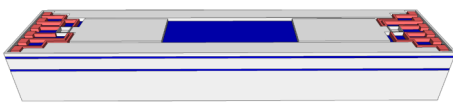

(c)

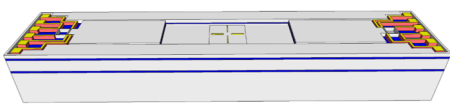

(e)

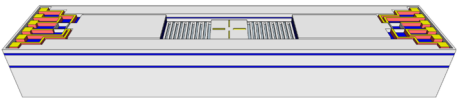

(f)

p-type Silicon

Buried oxide

p -type Silicon

Thin oxide layer

Chromium/Gold (Cr/Au)

Figure 4. Schematic representation of fabrication process of proposed device: dicing the SOI wafer into smaller pieces and cleaning (a), growing of thin oxide layer for boron diffusion (b), etching process of WB structures (c), metal deposition on contact area (d), dry etching of device front (e) and back sides (f).
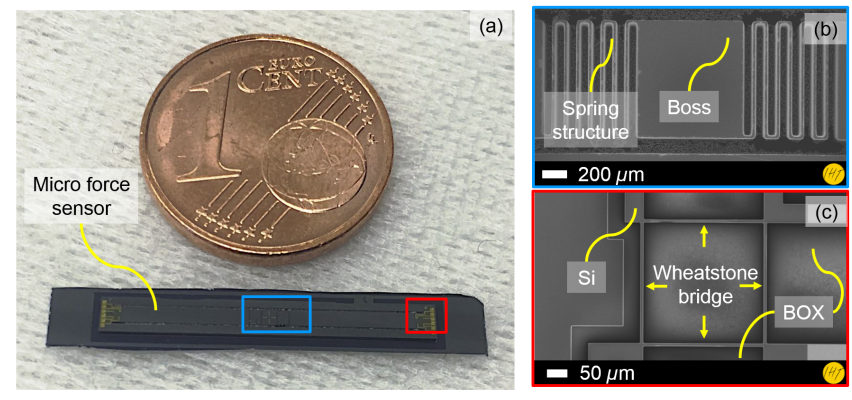

Figure 5. Fabricated micro force sensor (a). Magnified view of boss and meander-spring structures (b) and WB structures clamped on both device ends (c).

\section{Micro-fabrication}

Micro force sensors (design 1) were fabricated using a $p$ type double-layer silicon on insulator (DL-SOI) wafer with $<100>$ orientation and a resistivity of $0.01-0.02 \Omega \mathrm{cm}$ (Active Business Company $\mathrm{GmbH}$, Germany). One major advantage of using DL-SOI wafers is that a defined layer thicknesses can be selected. In this case, we have utilized a DLSOI wafer with a top layer (device layer), middle layer and bottom layer (handle layer) of $(3 \pm 1),(25 \pm 0.5)$ and $(350 \pm 15) \mu \mathrm{m}$, respectively. Furthermore, an oxide layer was buried between device and middle layers (BOX1), as well as between middle and handle layers (BOX2). The thick-

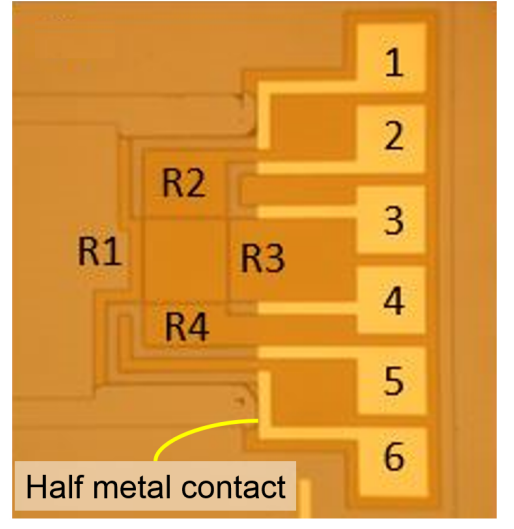

Figure 6. Optical micrograph of WB of design 1 with "half metal" contact.

nesses of these layers were $(0.2 \pm 0.01) \mu \mathrm{m}$ (BOX1) and $(0.5 \pm 0.025) \mu \mathrm{m}(\mathrm{BOX} 2)$. The use of DL-SOI for design 1 provides better control of the spring and WB structures with respect to the geometrical and doping uniformity. Using this novel approach, the etched WB structures were realized, which are electrically insulated from the underlying spring by the upper buried oxide layer. Thereby, current leakage can be avoided, which occurs by using implanted $p$-type resistor in $n$-type bulk silicon as in case of design 2 . However, higher 
wafer cost compared to standard bulk silicon material may be set against the use of DL-SOI.

To begin the sensor fabrication, a 4 " DL-SOI wafer was cut into $26 \mathrm{~mm} \times 26 \mathrm{~mm}$ pieces. Afterwards, the wafer was put into piranha solution $\left(\mathrm{H}_{2} \mathrm{O}_{2}: \mathrm{H}_{2} \mathrm{SO}_{4}=1: 1\right)$ and boiled at $90^{\circ} \mathrm{C}$ within $5 \mathrm{~min}$ to remove organic contamination on the surface (Fig. 4a). This step was performed prior to and after oxidation and before photolithography followed by producing $\sim 300 \mathrm{~nm}$ thermal oxide on the device layer. Subsequently, a oxide film on a particular contact position was etched and $p^{+}$diffusion (boron) was performed to obtain a high-quality contact formation (Fig. 4b). In contract to the WB of design 2, which was prepared by using of implanted piezoresistors, WB structures of design 1 were anisotropically created using ICP DRIE process utilizing $\mathrm{O}_{2}$ and $\mathrm{SF}_{6}$ as etch gases at a cryogenic temperature (Sentech Instruments GmbH, Germany; Wasisto et al., 2012, 2013, 2014). In this case, an etching parameter set of an ICP power of $500 \mathrm{~W}$, a high-frequency power of $6 \mathrm{~W}$, an $\mathrm{O}_{2}$ flux of $7 \mathrm{sccm}$ ( $\mathrm{sccm}$ is standard cubic centimeter per minute) and an $\mathrm{SF}_{6}$ flux of $129 \mathrm{sccm}$ at $-80^{\circ} \mathrm{C}$ was utilized (Fig. 4c). Once the WB structures had been fabricated, the oxide layer (BOX1) on the middle of the sensor was removed using a buffered hydrofluoric acid (HF; 6-7\%). Following this treatment, a "halfmetal" contact (i.e., metal layer was not deposited on the membrane) was realized on the device with $30 \mathrm{~nm}$ chromium and $300 \mathrm{~nm}$ gold by means of a lift-off process (Figs. 4d, 6). In the next step, photolithography was performed prior to etching of the middle layer. An etching duration of 12$15 \mathrm{~min}$ was needed for structuring the middle layer. Thereby, the boss structure was created and the front side of meander structures was defined. The removal of the second oxide layer (BOX2) was carried out by buffered HF (Fig. 4e). The most crucial step during the whole fabrication process was the back-side structuring of the meander spring. A $300 \mu \mathrm{m}$ deep etching of micro-structures with small gaps (i.e., $50 \mu \mathrm{m}$ ) was performed within $\sim 1.5 \mathrm{~h}$ using an ICP power of $500 \mathrm{~W}$, a high-frequency power of $7 \mathrm{~W}$, and a mixture of etch gases of $\mathrm{O}_{2}(6 \mathrm{sccm})$ and $\mathrm{SF}_{6}(129 \mathrm{sccm})$ (Fig. $\left.4 \mathrm{f}\right)$.

After completing the fabrication steps, a total of six micro force sensors with chip dimensions of $20 \mathrm{~mm} \times 2 \mathrm{~mm}$ were obtained from a $26 \mathrm{~mm} \times 26 \mathrm{~mm}$ DL-SOI sample (Fig. 5a). Evaluation in scanning electron microscope (SEM) was performed to analyze the dimensional stability of the realized WB and meander structures. The realized gaps between the meander-spring structures were larger than the designed ones (i.e., around $\sim 60 \mu \mathrm{m}$ ) due to overetching (Fig. $5 \mathrm{~b}$ ). However, this deviation was not crucial to the sensor performance in general (Hamdana et al., 2016). Moreover, this irregularity can be eliminated with fine tuning of the etch recipe, especially regarding the distribution of the process temperature. Furthermore, WB structures show only a small deviation of $(7.20 \pm 0.10) \mu \mathrm{m}$ (i.e., below $3 \%$ ) from each other (Fig. 5c). Hence, a low offset voltage $V_{\text {off }}$ (i.e., below $10 \mathrm{mV} \mathrm{V}^{-1}$ ) was

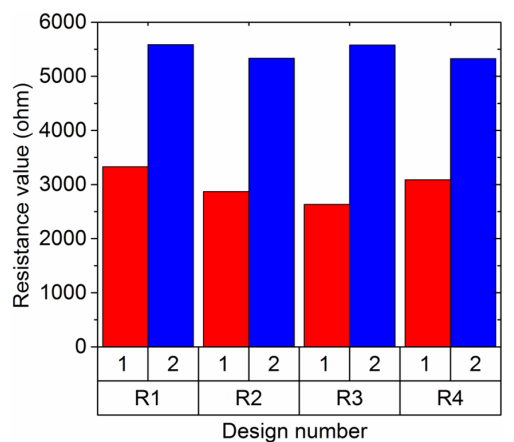

Figure 7. Measured resistance values of WBs comparing designs 1 and 2.

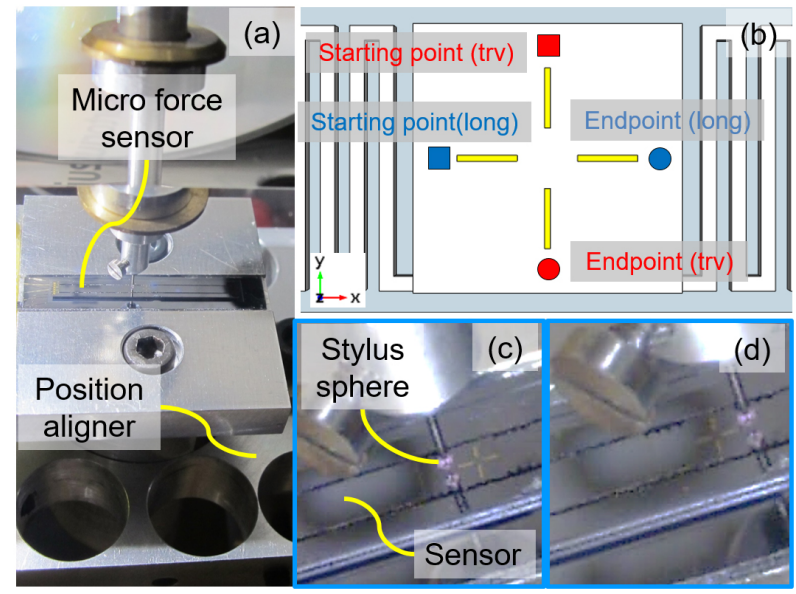

Figure 8. Measurement setup for calibrating micro force sensors (a). Different loading points were selected along two perpendicular lines (along $x$ axis: long.; along $y$ axis: trv.) across the center of the boss (b). Starting and end points of scanning procedure in longitudinal and transverse directions are shown in (c) and (d).

expected, which is very acceptable for the signal processing of the WB.

\section{Device characterizations}

To determine electrical properties of the sensor, measurements of WB resistances were carried out. The WB resistor arrangement of the sensor (design 1) was shown in Fig. 6. The bridge resistances of both designs were measured under zero applied load to the probing. As shown in Fig. 7, design 1 shows a value of $(2979 \pm 297) \Omega$ compared to design 2 with a higher resistance but lower standard deviation of $(5456 \pm 146) \Omega$. Furthermore, offset voltage values of both devices were also determined. In this case, design 1 has an offset value of $(0.05 \pm 0.01) \mathrm{mV} \mathrm{V}^{-1}$, whereas design 2 exhibits a higher offset value of $(23.15 \pm 0.008) \mathrm{mV} \mathrm{V}^{-1}$. The differences of resistor and offset voltage values may be due to material properties and fabrication processes of those two- 

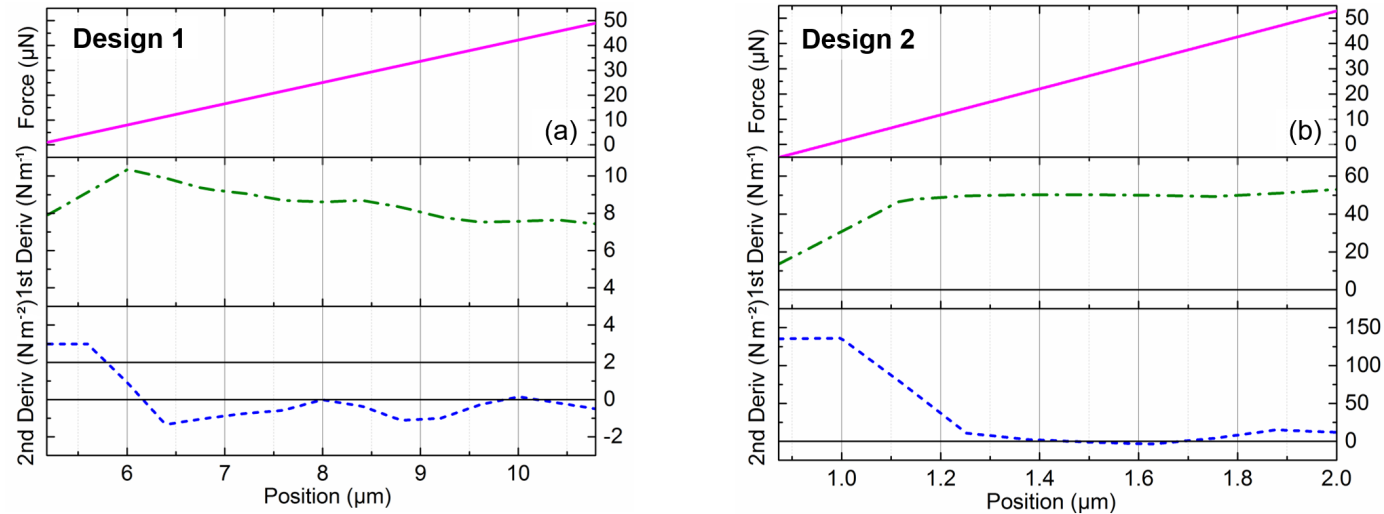

Figure 9. Non-linearity curves of design 1 with meander structure (a) and design 2 with combined micro-structures as spring (b).

Table 3. Direct comparison sensor properties of designs 1 and 2.

\begin{tabular}{lrrr}
\hline Parameter & Required & Design 1 (DL-SOI) & Design 2 $(p$-doped WB) \\
\hline Chip area & $<20 \mathrm{~mm} \times 20 \mathrm{~mm}$ & $20 \mathrm{~mm} \times 2 \mathrm{~mm}$ & $20 \mathrm{~mm} \times 2 \mathrm{~mm}$ \\
Probing area & $>1 \mathrm{~mm} \times 1 \mathrm{~mm}$ & $2 \mathrm{~mm} \times 2 \mathrm{~mm}$ & $2 \mathrm{~mm} \times 2 \mathrm{~mm}$ \\
Resonance frequency & $>100 \mathrm{~Hz}$ & $805 \mathrm{~Hz}$ & $805 \mathrm{~Hz}$ \\
Noise at bandwidth of $10 \mathrm{~Hz}$ & $<0.1 \mu \mathrm{N}$ & $0.5 \mu \mathrm{V} / 0.03 \mu \mathrm{N}^{*}$ & $0.33 \mu \mathrm{V} / 0.07 \mu \mathrm{N}$ \\
Measurement range & $1 \mathrm{mN}$ & $50 \mu \mathrm{N}$ & $50 \mu \mathrm{N}$ \\
Typical wafer price per pcs $\left(4^{\prime \prime}, 10 \mathrm{pcs}\right)$ & - & around EUR 400 & around EUR 25 \\
\hline
\end{tabular}

* Measured at WB structures.

types of WB structures. In addition, cross-sensitivities of the fabricated WBs against light, temperature and moisture were determined at a WB supply voltage of $1 \mathrm{~V}$. For measurements under direct illumination with a specific wavelength of $635 \mathrm{~nm}$, a high power cold-light source (KL 1500, Schott AG, Germany) and an optical power meter (Thorlabs GmbH, Germany) were used. Measurements under controlled temperature and moisture were performed in a sealed chamber. Assuming controlled measuring room conditions (i.e., illuminance level $<104$ lumen $\mathrm{m}^{-2}$, temperature drift $<1 \mathrm{~K}$ and relative humidity change $<6 \%$ ), we observed changes of offset voltage less than $1 \mu \mathrm{V}$ corresponding to force errors below $0.1 \mu \mathrm{N}$.

During the force application procedure, the sensor was mounted on an aluminum holder of a three axis nanopositioning system. Precise sensor movement with a reproducibility of $5 \mathrm{~nm}$ and at resolution of $1 \mathrm{~nm}$ was performed against a probing body with a $300 \mu \mathrm{m}$ ruby sphere, which was mounted on the pan of a compensation balance (Fig. 8a). Thus, the resulting force on the probing area was controlled at a resolution of $1 \mathrm{nN}$ and a reproducibility of $2.5 \mathrm{nN}$ (Peiner and Doering, 2005). Sensor performance was assessed based on calibration on different probing positions on boss structure, i.e., along the $x$ axis (longitudinal) and the $y$ axis (transverse). Total length and increment of 500 and $50 \mu \mathrm{m}$ were selected during measurements in both longitudinal (long) and transverse (trv) directions, respectively (Fig. 8b-d).
Turning now to the force application procedure, the sensor was fixed on an aluminum holder, which was mounted on a nano-positioning unit. This part can be precisely moved in 3-D directions with a maximum displacement of $100 \mu \mathrm{m}$ and a resolution of $1 \mathrm{~nm}$. The contact to the sensor was then realized using a stylus with a glued ruby sphere with diameter of $300 \mu \mathrm{m}$ (Fig. 8a). For both designs, a maximum force of $50 \mu \mathrm{N}$ was applied and controlled by a compensation balance. Measured values were collected in steps of $400 \mathrm{~nm}$ movement along the $z$ axis. In this work, we assessed the sensor responses on force applications at different positions on the probing body (boss). Therefore, the load was applied along the longitudinal axis (i.e., $x$ axis) and the transverse axis (i.e., $y$ axis) through the center of the boss. Between the measurement points (i.e., position 1 and position 2), increment and maximum longitudinal position changes of 50 and $500 \mu \mathrm{m}$ were used, respectively (Fig. 8b-d).

As shown in Fig. 9a-b, both sensor designs demonstrate good linearity up to $50 \mu \mathrm{N}$. Regarding their structural flexibility, design 1 indicates a more compliant behavior with a greater deflection value of boss in the $z$ axis than design 2 . Consequently, the first and second derivatives of the forcedeflection curve of design 2 exhibit greater values, which indicate the need of higher force to achieve the same deflection. This attribute could be unfavorable for material stability and signal processing. Nevertheless, mechanical properties and sensor stability were investigated by monitoring sen- 

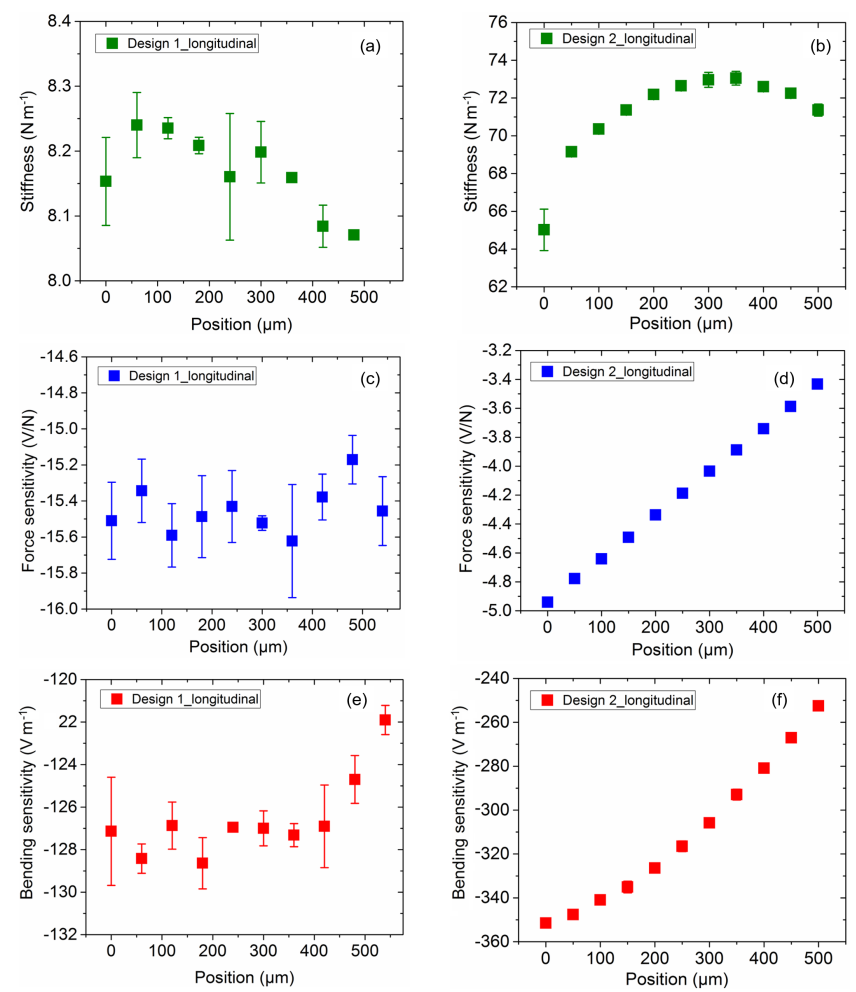

Figure 10. Results of sensor calibration procedure (maximum applied force: $50 \mu \mathrm{N}$ ) at different loading points on the boss structure in longitudinal direction: stiffness $(\mathbf{a}, \mathbf{b})$, force sensitivity $(\mathbf{c}, \mathbf{d})$ and bending sensitivity $(\mathbf{e}, \mathbf{f})$.

sor responses during increasing and decreasing loading. The term increasing loading (i.e., loading) referred to measurement state, when the stylus first contacted the boss until its final lower position. On the contrary, decreasing loading (i.e., unloading) referred to boss movement from a lower position to its initial position under a zero applied load. From repeated measurements of both motions, three important device properties (i.e., stiffness, force sensitivity and bending sensitivity) can be extracted, and the performances of different sensor designs can be evaluated.

Mean device stiffness derived from loading and unloading of different sensor designs are illustrated in Fig. 10a and $b$. As predicted in the FEM simulations, design 1 exhibits more compliant behavior than design 2 . With a mean stiffness value in longitudinal $(8.15 \pm 0.08) \mathrm{N} \mathrm{m}^{-1}$ and transverse $(8.21 \pm 0.06) \mathrm{N} \mathrm{m}^{-1}$ directions, the maximum stiffness deviations along the probing area (boss) are below $2 \%$ from the mean value (Figs. 10a, 11a). On the other side, design 2 yields a higher mean stiffness $(70.57 \pm 2.58)$ (longitudinal) and $(71.66 \pm 1.26) \mathrm{N} \mathrm{m}^{-1}$ (transverse) with a stiffness deviation up to $\sim 8 \%$ from the mean value (Figs. 10b, 11b). This means that design 1 can be deflected with lower force and smaller increments between forces and being less affected by the loading position than design 2 . Combining this advan-
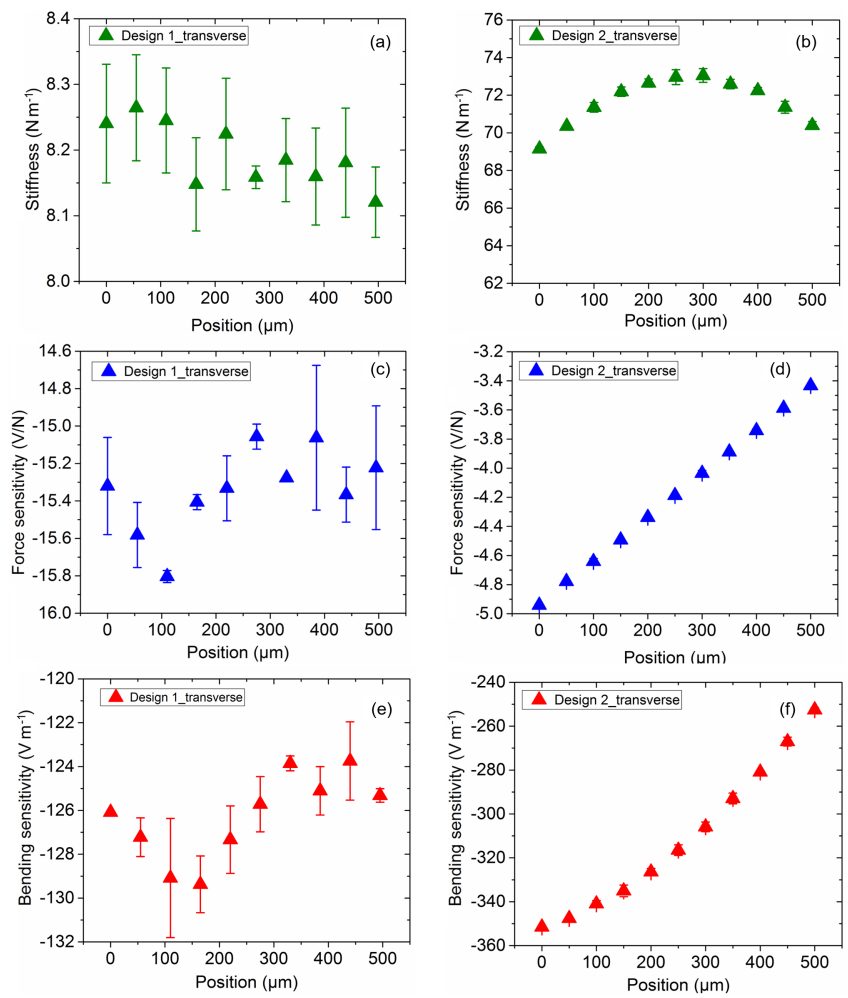

Figure 11. Results of sensor calibration procedure (maximum applied force: $50 \mu \mathrm{N}$ ) at different loading points on the boss structure in transverse direction: stiffness $(\mathbf{a}, \mathbf{b})$, force sensitivity $(\mathbf{c}, \mathbf{d})$ and bending sensitivity $(\mathbf{e}, \mathbf{f})$.
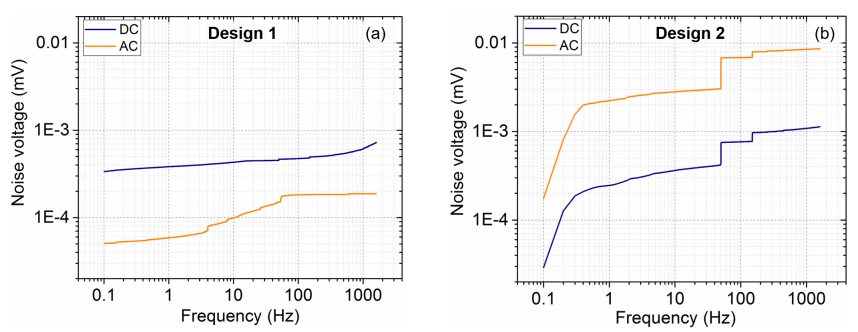

Figure 12. Electrical noise performance of micro force sensors applied without (DC) or with (AC) carrier frequency of an SOI-based WB (a) compared to implanted (b) WB.

tage with sensitivity its high measured mean $(15.45 \pm 0.17)$ and (15.34 \pm 0.25$) \mathrm{V} \mathrm{N}^{-1}$ in longitudinal and transverse directions, respectively and a maximum deviation value of $\sim 3 \%$, the design 1 shows better performance to measure small forces then the design 2 with a maximum deviation in sensitivity of around $\sim 39 \%$ (Figs. 10c-d, 11c-d). In terms of bending sensitivity, design 1 yielded mean values in both longitudinal and transverse directions, i.e., $(126.59 \pm 2.10)$ and $(126.29 \pm 2.11) \mathrm{V} \mathrm{m}^{-1}$, with a maximum deviation of $\sim 4 \%$. In contrast, design 2 exhibited bending sensitivity values of $(304.47 \pm 38.60)$ and $(310.66 \pm 33.67) \mathrm{V} \mathrm{m}^{-1}$ with deviations of $\sim 33 \%$ (Figs. 10e-f, 11e-f). Tables 2 and 3 
summarize the measurement results on both micro force sensor designs together with the requirements. Therefore, specified parameters of both designs were confirmed experimentally. However, the measurement range is limited between 0 and $50 \mu \mathrm{N}$, which has to be extended to $1 \mathrm{mN}$.

The minimal detectable force of the sensor is limited by the effective noise voltage of the WB. In comparison to a WB with implanted resistances, the SOI-based bridges are electrically isolated to the surrounded substrate by a BOX layer. This allows us to operate it at an AC voltage with no current leakage. For the measurements, we used the MGC plus measuring amplifier system of Hottinger Baldwin Messtechnik (HBM) GmbH with the insert modules ML10B for DC supply and the ML30B for AC supply. The ML30B uses a carrier frequency of $600 \mathrm{~Hz}$. Figure 12a shows the measured noise voltage of the SOI-based micro force sensor (i.e., design 1) with DC and AC supplies. In this case, using an AC carrier frequency, the noise voltage of the micro force sensor in the frequency ranging from 0.1 to $1 \mathrm{kHz}$ could be reduced by a factor of 3. In contrast, the $p-n$ junctions of the implanted WBs are massively affected by the potential change between $p$-type WB and the n-doped substrate in case of AC supply to the WB. Figure $12 \mathrm{~b}$ depicts the noise voltage of an implanted bridge (i.e., design 2) with and without carrier frequency. Applying DC voltage during the measurements, noise voltages varied from 0.1 to $50 \mathrm{~Hz}$ have shown more or less the same values. Meanwhile, when the AC voltage was supplied to the bridges, the noise values are $>20$ times higher than those at DC supply.

\section{Conclusions}

This study has set out to measure and assess a developed meander-type sensor for calibration of nano-indenters in the micro-Newton force range compared to an industrially manufactured device. Finite element modeling (FEM) was performed to predict and to provide better understanding of sensor response. The impact on different spring structures (i.e., meander structure for design 1 and combined microstructures of design 2) was investigated through the evaluation of the device properties using FEM and fabricated sensor. Furthermore, repeated measurements by applying vertical loads at different contact points along the $x$ and $y$ axes across the probing body (boss) were performed. While both designs show good measurement linearity, the new one (design 1) has a more flexible structure with higher sensitivity than the industrially manufactured device (design 2). Moreover, design 1 shows an improved behavior concerning the dependence of measured force and deflection on the loading position of the sensor. The loading position can be changed within a range of $\pm 250 \mu \mathrm{m}$ and force and deflection sensitivity do not change by more than $3 \%$. Furthermore, it has been shown that double-layer silicon on insulator (DL-SOI) material provides better sensor performance in terms of electrical noise. These characteristics will have implications for providing transferable force standards with excellent functionality and high reliability for the micro-Newton scale. 


\section{Biographies}

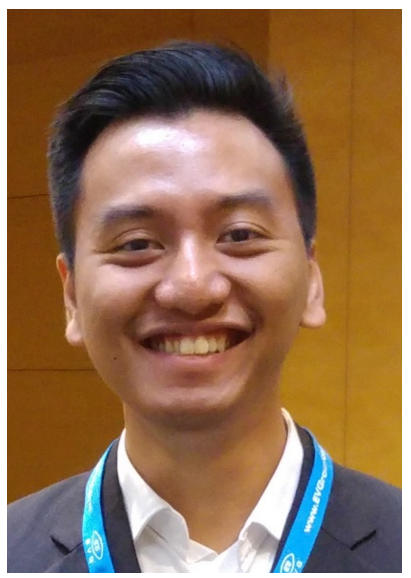

Gerry Hamdana received his Bachelor of Engineering degree in mechanical engineering from the Esslingen University of Applied Science in 2012 and Master of Science degree in mechanical engineering specializing in mechatronic/microsystems technology from the Braunschweig University of Technology (TU Braunschweig), Germany, in 2015. Currently, he is pursuing his $\mathrm{PhD}$ at the Institute of Semiconductor Technology (IHT), TU Braunschweig, Germany. His research interests include semiconductor processing, microsystems technology and micro/nano-electromechanical systems (MEMSs/NEMSs).

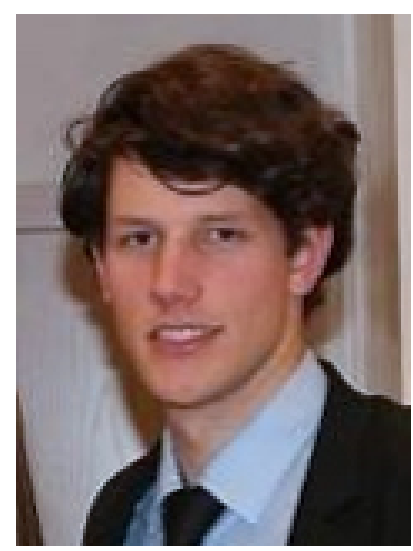

Maik Bertke received the Bachelor of Science degree and the Master of Science degree in electrical engineering from the Technische Universität Braunschweig, Germany, in 2013 and 2016, respectively. Currently, he is working towards the $\mathrm{PhD}$ at the Institute of Semiconductor Technology (IHT), TU Braunschweig and Laboratory for Emerging Nano-metrology, Germany, where his main interests are in the fields of micro-/nano-electromechanical system (MEMS/NEMS)-based sensors.

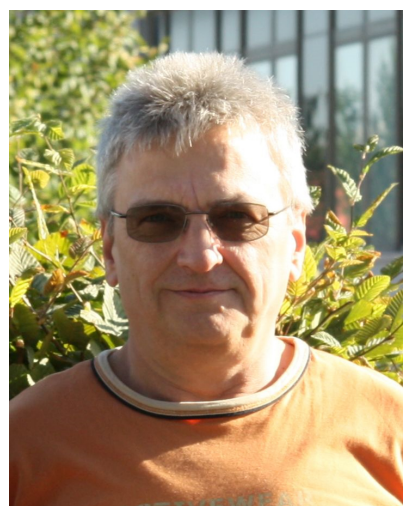

Lutz Doering received a Dr.-Ing. degree in micro-electronics technology, precision instrument technology and computer science from the Dresden University of Technology, Dresden, Germany, in 1990. He joined the Department 5.1 Nano- and Micro-metrology, Physikalisch-Technische Bundesanstalt, Braunschweig, Germany, in 2001. His current research interests include characterization and optimization of micromachined cantilever sensors designed for measuring coordinate and roughness parameters and for the transfer of the micro- and nano-Newton force standard to tactile probing tools.

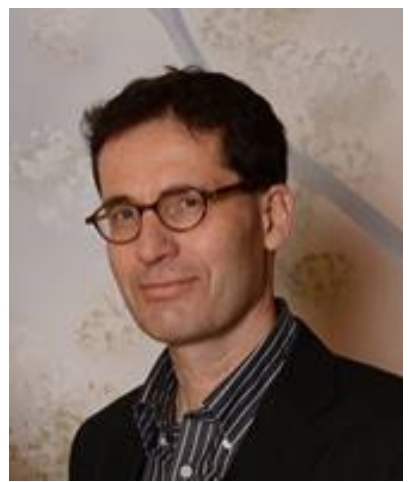

Thomas Frank received a Dr.-Ing. degree in mechanical engineering, microtechnology at the Technical University of Ilmenau, Ilmenau, Germany, in 2002. Until 2008, he was Managing Director of Little Things Faktory GmbH in Ilmenau. Since 2009 he has been manager of the business unit MEMSs of the CiS Research Institute for Mikrosensorik $\mathrm{GmbH}$ in $\mathrm{Er}$ furt, Germany. The range of his tasks includes silicon-based piezoresistive sensors for pressure, force, hardness, geometry and roughness. 


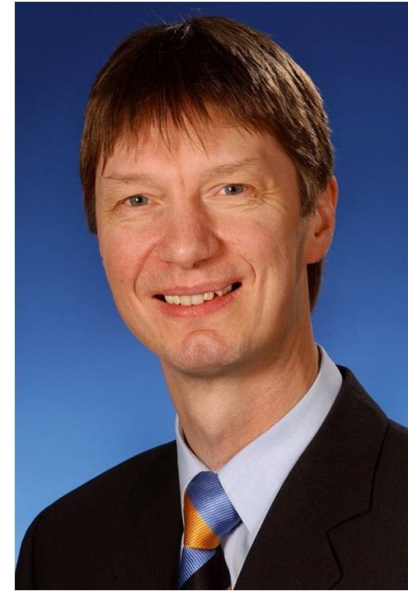

Uwe Brand received a $\mathrm{PhD}$ in physics from the $\mathrm{TU}$ Braunschweig, Germany, in 1991. Since 2000, he has been the head of the working group 5.11 Hardness and Tactile Probing Methods in German National Metrology Institute, Physikalisch-Technische Bundestanstalt (PTB), Braunschweig, Germany. His main research interests include the further development of the nano-indentation technique and standard devices. His research group creates the basis for the traceability of hardness measurements in industry, research and calibration laboratories to the national standards.

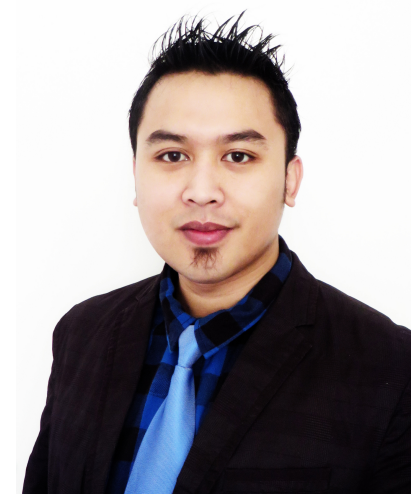

Hutomo Suryo Wasisto received the Bachelor of Engineering degree in electrical engineering (Cum Laude) from the Gadjah Mada University, Indonesia, the Master of Engineering degree in semiconductor engineering (Cum Laude) from the Asia University, Taiwan, and the Doktor-Ingenieur (Dr.Ing.) degree in electrical engineering (Summa Cum Laude) from the Technische Universität Braunschweig (TU Braunschweig), Germany in 2008, 2010 and 2014, respectively. He was a postdoctoral research fellow at the School of Electrical and Computer Engineering (ECE), Georgia Institute of Technology, Atlanta, USA, in 2015-2016. Since 2016, he has been Head of Optoelectromechanical Integrated Nano-systems for Sensing (OptoSense) Group in the Laboratory for Emerging Nano-metrology (LENA), Braunschweig, Germany. His main research interests include nano- opto-electro-mechanical systems (NOEMS), nano-sensors, nano-electronics, nano-LEDs, nano-generators and nanometrology. He has published more than 60 papers in international scientific peer-reviewed journals and conference proceedings as well as two European and German patents with more than 200 citations. Hutomo Suryo Wasisto has also been the recipient of the best-paper award and the best young scientist award at the 8th IEEE International Conference on Nano-/Micro-Engineered and Molecular Systems (IEEE NEMS 2013) in Suzhou, China, and the 26th European Conference on Solid-State Transducers (Eurosensors 2012) in Krakow, Poland. In 2014, he received the WalterKertz-Studienpreis (Walter Kertz Study Award) for his excellent doctoral dissertation and achievements of scientific studies at the interface between physics, electrical engineering and information technology from the TU Braunschweig, Germany. In 2015, he has been awarded with the Transducers 2015 travel grant award from Transducer Research Foundation (TRF), USA, in the 18th International Conference on Solid-State Sensors, Actuators and Microsystems (Transducers 2015), Anchorage, Alaska USA. Since 2013, he has been a reviewer for more than 20 scientific journals and international research organizations (e.g., IEEE Journal of Micro-electromechanical Systems, IEEE Sensors Journal, IEEE Transactions on Industrial Electronics, IOP Journal of Micro-mechanics and Micro-engineering, Sensors and Actuators A: Physical, International Journal of Electronics, Sensors, Applied Surface Science, and Journal of Hazardous Materials).

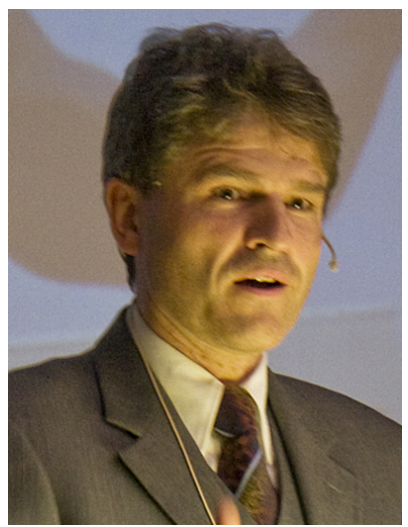

Erwin Peiner received $\mathrm{PhD}$ degrees in metastable binary metal compounds by ion beam mixing from the University of Bonn, Bonn, Germany, in 1988, and the Venia Legendi degree in semiconductor technology from the Faculty of Mechanical and Electrical Engineering, TU Braunschweig, Braunschweig, Germany, in 2000. Currently, he is the leader and professor of the Semiconductor Sensors and Metrology Group at IHT, TU Braunschweig. He has published more than 250 papers in international journals and conference pro- 
ceedings. He is the project coordinator of the collaborative project "HmtS" funded by the BMBF.

\section{Data availability}

Research data are available upon request to the authors.

Competing interests. The authors declare that they have no conflict of interest.

Acknowledgements. The authors thank Juliane Breitfelder and Doris Rümmler for their technical support. The first author gratefully acknowledge support by Braunschweig International Graduate School of Metrology (B-IGSM). This work was performed in the collaborative project "Hochgeschwindigkeitsmikrotaster für die Messung an Oberflächen von Strukturen mit großem Aspektverhältnis (HmtS)" funded by German Federal Ministry of Education and Research (BMBF) under no. 03V0409.

Edited by: G. Gerlach

Reviewed by: two anonymous referees

\section{References}

Bonev, I. and Zlatanov, D.: Advantages of the modified Euler angles in the design and control of PKMs, Proceeding Parallel Kinematic Mach. Int. Conf., 171-1880, 2002.

Brand, U., Li, Z., Gao, S., Hahn, S., and Hiller, K.: Silicon double spring for the simultaneous calibration of probing forces and deflections in the micro range, Meas. Sci. Technol., 27, 15601, doi:10.1088/0957-0233/27/1/015601, 2016.

Choi, W., Lee, J., Yoo, Y. K., Kang, S., Kim, J., and Lee, J. H.: Enhanced sensitivity of piezoelectric pressure sensor with microstructured polydimethylsiloxane layer Enhanced sensitivity of piezoelectric pressure sensor with microstructured polydimethylsiloxane layer, Appl. Phys. Lett., 104, 123701, doi:10.1063/1.4869816, 2016.

COMSOL Multyphisics: COMSOL Multiphysics Release Notes: Version 4.3b, COMSOL AB, Stockholm, Sweden, available at: https://www.comsol.de/shared/downloads/4.3b/ReleaseNotes. pdf, 2013.

Doering, L., Nesterov, V., and Peiner, E.: Mikrokraftsensor, German Patent No. 102010012 701, 2013.

Frühauf, J., Gärtner, E., Herrmann, K., Menelao, F., Schwenk, D., Chudoba, T., and Vollmar, H.: Calibration of Instruments for Hardness Testing, Recent Adv. Theory Pract. Hardness Meas. HARDMEKO, 141, 2007a.

Frühauf, J., Gärtner, E., and Herrmann, K.: Standard for Calibration of Instruments of Hardness Testing, Tech. Mess., 74, 7-8, doi:10.1524/teme.2007.74.7-8.385, 2007b.

Fu, K., Chang, L., Zheng, B., Tang, Y., and Wang, H.: On the determination of representative stress - strain relation of metallic materials using instrumented indentation, J. Mater., 65, 989-994, doi:10.1016/j.matdes.2014.10.018, 2015.
Hamdana, G., Wasisto, H. S., Doering, L., Yan, C., Zhou, L., Brand, U., and Peiner, E.: Double-meander spring silicon piezoresistive sensors as microforce calibration standards, Opt. Eng., 55, 91409, doi:10.1117/1.OE.55.9.091409, 2016.

Hopcroft, M. A., Nix, W. D., and Kenny, T. W.: What is the Young's modulus of silicon?, J. Microelectromech. S., 19, 229238, doi:10.1109/JMEMS.2009.2039697, 2010.

Kähler, J., Stranz, A., Doering, L., Merzsch, S., Heuck, N., Waag, A., and Peiner, E.: Fabrication, packaging, and characterization of p-SOI Wheatstone bridges for harsh environments, Microsyst. Technol., 18, 869-878, doi:10.1007/s00542-011-1396-6, 2012.

Kähler, J., Peiner, E., Stranz, A., and Waag, A.: High temperature piezoresisitive strain gauges made of Silicon-On-Insulator, United States Patent application, US 2013/0068008 A1, WO 2012/141843 A3, 2013.

Lee, J., Choi, W., Yong, Y. Kyoung, Hwang, K. Seon, Lee, S. M., Kang, S., Kim, J., and Lee, J. Hoon: A micro-fabricated force sensor using an all thin film piezoelectric active sensor, Sensors, 14, 22199-22207, doi:10.3390/s141222199, 2014.

Li, Z. and Brand, U.: Towards Quantitative Characterisation of the Small Force Transducer Used in Nanoindentation Instruments, Modern Instrumentation, 61-67, 2013.

Mehrtash, M., Zhang, X., and Khamesee, M. B.: Bilateral Magnetic Micromanipulation Using Off-Board Force Sensor, IEEE/ASME Trans. Mechatronics, 20, 3223-3231, doi:10.1109/TMECH.2015.2417116, 2015.

Merzsch, S., Steib, F., Wasisto, H.S., Stranz, A., Hinze, P., Weimann, T., Peiner, E., and Waag, A.: Production of vertical nanowire resonators by cryogenic-ICP-DRIE, Microsystem technologies, 20, 759-767, doi:10.1007/s00542-013-2032-4, 2014.

Mizutani, Y.: Disturbance Compensation based on Data Memory for Macro - Micro Bilateral Control, IEEE Int. Symp. Ind. Electron., 2013.

Mizutani, Y.: Analysis, Modeling, and Compensation of Friction for Scaled Bilateral Control, IEEJ J. Ind. Appl., 3, 344-349, 2014.

Mohan, N., Luo, Y., and Shen, Y.: Developing a Self-Powered and Directly Digitized Piezoelectric Micro Sensor for Monitoring Blood Pressure Change inside Brain Aneurysm after Endovascular Treatment?: A Feasibility Study, 5th Annu. IEEE Int. Conf. Cyber Technol. Autom. Control Intell. Syst., 2115-2120, doi:10.1109/CYBER.2015.7288275, 2015.

Nili, H., Kalantar-zadeh, K., Bhaskaran, M., and Sriram, S.: Progress in Materials Science In situ nanoindentation?: Probing nanoscale multifunctionality, Prog. Mater. Sci., 58, 1-29, doi:10.1016/j.pmatsci.2012.08.001, 2013.

Ousaid, A. M., Haliyo, D. S., Regnier, S., and Hayward, V.: A Stable and Transparent Microscale Force Feedback Teleoperation System, IEEE/ASME Trans. Mechatronics, 20, 2593-2603, doi:10.1109/TMECH.2015.2423092, 2015.

Park, S. J., Doll, J. C., and Pruitt, B. L.: Piezoresistive cantilever performance part I: Analytical model for sensitivity, J. Microelectromech. S., 19, 137-148, doi:10.1109/JMEMS.2009.2036581, 2010.

Peiner, E. and Doering, L.: Force calibration of stylus instruments using silicon microcantilevers, Sensor. Actuat.-A Phys., 123124, 137-145, doi:10.1016/j.sna.2005.02.031, 2005.

Viry, L., Levi, A., Totaro, M., Mondini, A., Mattoli, V., Mazzolai, B., and Beccai, L.: Flexible three-axial force sensor for soft 
and highly sensitive artificial touch, Adv. Mater., 26, 2659-2664, doi:10.1002/adma.201305064, 2014.

Wasisto, H. S., Merzsch, S., Waag, A., Uhde, E., Salthammer, T., and Peiner, E.: Airborne engineered nanoparticle mass sensor based on a silicon resonant cantilever, Sensor. Actuat. B-Chem., 180, 77-89, doi:10.1016/j.snb.2012.04.003, 2012.

Wasisto, H. S., Merzsch, S., Stranz, A., Waag, A., Uhde, E., Salthammer, T., and Peiner, E.: Femtogram aerosol nanoparticle mass sensing utilising vertical silicon nanowire resonators, Micro Nano Lett., 8, 554-558, doi:10.1049/mnl.2013.0208, 2013.

Wasisto, H. S., Huang, K., Merzsch, S., Stranz, A., Waag, A., and Peiner, E.: Finite element modeling and experimental proof of NEMS-based silicon pillar resonators for nanoparticle mass sensing applications, Microsyst. Technol., 20, 571-584, doi:10.1007/s00542-013-1992-8, 2014.

Wasisto, H. S., Merzsch, S., Uhde, E., Waag, A., and Peiner, E.: Handheld personal airborne nanoparticle detector based on microelectromechanical silicon resonant cantilever, Microelectron. Eng., 145, 96-103, doi:10.1016/j.mee.2015.03.037, 2015a.
Wasisto, H. S., Merzsch, S., Uhde, E., Waag, A., and Peiner, E. Partially integrated cantilever-based airborne nanoparticle detector for continuous carbon aerosol mass concentration monitoring, J. Sens. Sens. Syst., 4, 111-123, doi:10.5194/jsss-4-111-2015, 2015. 2015 b.

Wasisto, H. S., Doering, L., Daus, A., Brand, U., Frank, T., and Peiner, E.: Development of silicon microforce sensors integrated with double meander springs for standard hardness test instruments, Proc. SPIE 9517, Smart Sensors, Actuators, and MEMS VII, and Cyber Physical Systems, 95171X (21 May 2015), doi:10.1117/12.2180117, 2015c.

Woo, S.-J., Kong, J.-H., Kim, D.-G., and Kim, J.-M.: A thin allelastomeric capacitive pressure sensor array based on microcontact printed elastic conductors, J. Mater. Chem., 2, 4415, doi:10.1039/c4tc00392f, 2014.

Yetna Njock, M., Roudet, F., Idriss, M., Bartier, O., and Chicot, D.: Work-of-indentation coupled to contact stiffness for calc. elastic modulus by instrumented indentation, Mech. Mater., 94, 170 179, doi:10.1016/j.mechmat.2015.12.003, 2016. 